ORIGINAL ARTICLE

\title{
Study to Determine the Incidence of Allergic Fungal Sinusitis among Patients with Nasal Polyps and Its Related Risk Factors
}

\author{
BAKHT ZADA ${ }^{1}$, EJAZ AHMED ${ }^{2}$, MUHAMMAD HABIB ${ }^{3}$, ZAFAR IQBAL ${ }^{4}$, REHAN SALEEM ${ }^{5}$, MUHAMMAD TAYYAB \\ RASHEED 6 \\ ${ }^{1}$ ENT Head \& Neck Surgery, Lady Reading Hospital MTI, Peshawar \\ ${ }^{2}$ Assistant Professor ENT, Mohtarma Benazir Bhutto Shaheed Medical College (MBBSMC) \\ Div. Headquarter Teaching hospital, Mirpur \\ ${ }^{3}$ Associate professor ENT, Peshawar Medical College, Peshawar \\ ${ }^{4}$ Associate Professor ENT, Azra Nahid Medical College, Lahore \\ ${ }^{5}$ Senior Registrar ENT Department, Azra Nahid Medical College, Lahore \\ ${ }^{6}$ Assistant Professor ENT, Aziz Bhatti Shaheed Teaching Hospital/ Nawaz Sharif Medical College, Gujrat \\ Corresponding author: Dr. Muhammad Habib, Email: mhabibent@gmail.com, Cell No: +92 3005938475
}

\begin{abstract}
Aim: To govern the incidence of allergic fungal sinusitis in patients with nasal polyposis. Various anatomical risk factors were also investigated, including turbinate hypertrophy, deviated nasal septum and comorbidities such as asthma and diabetes.

Study Design: This is a Descriptive cross-sectional study.

Place and duration of study:The study was conducted at ENT Head \&Neck Surgery department, Lady Reading Hospital MTI, Peshawar and Azra Nahid Medical College, Lahore for the duration of six months from May 2020 to October 2020.

Methods: 110 patients with nasal polyps were evaluated and operated on. Samples were sent for histopathological examination and culture. All patients were assessed with clinical examination and detailed history. Laboratory tests were performed including complete blood counts, urea, electrolytes, ECG and chest radiographs for the suitability of general anesthesia as a prerequisite for surgery. In 95\% of cases, computed tomography was recommended to check for sinus involvement, bone erosion, osteo-hypertrophic complex, turbinate hypertrophy, nasal septal deviation, and intracranial and intra-orbital enlargement. MRI examinations were also recommended in cases of suspected intraocular and intracranial disease (5\%). Data was scrutinized on a computer using SPSS version 22.0.

Results:Of the 110 patients, 65 were male and 45 were female, with a mean age of 1 and ranged from 7 to 80 years. All patients had nasal polyps. The incidence of AFS was approximately $29.1 \%$ and the remaining 78 had a different pathology. Major deviation of the nasal septum and bilateral hypertrophy of the inferior turbinate's were observed in $6(18.7 \%)$ and $4(12.5 \%)$ patients, respectively. Unilateral nasal polyps were observed in 7 (21.9\%) patients and bilateral nasal polyps in 18 (52.3\%). 19/32 (59.4\%) of the cases underwent functional endoscopic surgery of the paranasal sinuses, and in 6 (18.7\%) external fronto-ethmoidectomy. Two patients underwent nasal ethmoidectomy. Septoplasty and endoscopic sinus surgery were performed in a total of $3(9.37 \%)$ cases.
\end{abstract}

Key words:Allergic bronchopulmonary aspergillosis (ABPA), Allergic fungal sinusitis (AFS).

\section{INTRODUCTION}

In 1976, Safirstein noted that the combination of nasal polyps and sinus cultures that crust scab formation and aspergillosis share clinical similarities with allergic bronchopulmonary aspergillosis (ABPA). Over time, this ailment is recognized as allergic fungal sinusitis (AFS) ${ }^{1-2}$. Allergic fungal sinusitis is mostly considered a disease different chronic sinusitis. It is always associated with nasal polyps $^{3-4}$. Patients with chronic sinusitis are at a higher risk of allergic fungal sinusitis. Sociodemographic factors appear to have a significant impact on the incidence and incidence of allergic fungal sinusitis. The precise pathophysiology of the disease is unclear. Manning and hiscolleague's popular theoryaregrounded on the supposition that numerousinterconnected events and factorsresult in the persistence and development of the disease ${ }^{5-6}$. Primary, the atopic host is exposed to the fungus through normal nasal breathing, thenpreliminary antigenic stimulus begins. The type III (immune complex) and Gel-Coombs's type I (IgE)-mediated reaction induces a strong eosinophilic response of inflammation ${ }^{7}$. The subsequent inflammation causeobstacle of the orifices, which can be aggravated by certain anatomical factors, such as a distorted cartilage of the septal septum, and turbinate hypertrophy. As a result, media are preferred for fungal growth with obstruction of the sinus opening ${ }^{8-9}$. Thus, with increased exposure to antigens, this cycle results in the formation of allergic mucin, a material that seals the affected sinuses of a patient with AFS. Allergic mucin, a greenish to yellowish, dense, hard and very sticky substance, is often found during surgery and is considered a hallmark of the disease $e^{10-11}$. The diagnosis is based on the diagnostic criteria of Bent and Kuhan in 1994 and showed five common features: 1: Type I (IgE-mediated) hypersensitivity. 2: Nasal polyposis. 3: Characteristic results of radiological examinations. 4: Eosinophilic mucin 5: Positive coloring of fungi.

\section{Treatment options}

A: Medical Treatment: 1: Steroid Therapy. 2: Immunotherapy. 3: Antifungal therapy.

B: Surgical treatment: Complete disease elimination and marsupialization therapy are the cornerstones of treatment. Although much research has been conducted abroad on the morbidity and diagnosis of allergic fungal sinusitis, only 
a few bibliographic references are available in a local journal ${ }^{12}$. Hospital data reflect the severity of the disease and its prevalence in our population. Therefore, this study was conducted to determine the prevalence of allergic fungal sinusitis and risk factors associated with nasal polyps in our population.

\section{METHODS}

110 patients with nasal polyps were included in the study. After obtaining informed consent, patients referred from ENT Head \& Neck Surgery department, Lady Reading Hospital MTI, Peshawar and AzraNahid Medical College, Lahore for the duration of six months from May 2020 to October 2020. All subjects were assessed with clinical examination and detailed history. The clinical examination was performed by a resident and consultant using a nasal speculum and well-lit headlight, and the results were confirmed with a flexible or rigid endoscopic fiber providing direct visualization of the nasal cavity and associated pathology, such as a deviated septum or turbinate hypertrophy were documented. Laboratory tests were performed including complete blood counts, urea, electrolytes, ECG and chest radiographs for the suitability of general anesthesia as a prerequisite for surgery. In $95 \%$ of cases, computed tomography was recommended to check for sinus involvement, bone erosion, osteohypertrophic complex, turbinate hypertrophy, nasal septal deviation, and intracranial and intra-orbital enlargement. MRI examinations were also recommended in cases of suspected intraocular and intracranial disease (5\%). The study included patients who reported to the ENT clinic and diagnosed with nasal polyposis. Both men and women participated in this study. Diabetic and asthma patients with nasal polyps were also included in the study. Patients who refused surgery and biopsy were not included in this study. A surgical plan was established contingent on the disease extent, and a radiologist's opinion was obtained. General anesthesia was used in all cases. To minimize relapse, AFS treatment was targeted at removing nasal polyps, allergic mucin, and debris. Surgery can be performed using a variety of approaches and techniques. We typically use the following approaches: 1) Endoscopic sinus surgery. 2) Nasal ethmoidectomy. 3) External Ethmoidectomy. 4) Operation Caldwell Luc. After removal of disease, samples for histopathology were collected in formalin solution and the culture sample was transferred in a sterile normal saline bottle. After receiving the histopathological report and culture, the postoperative treatment plan was established. The final diagnosis was made on the basis of culture and histopathological reports. To simplify calculations and results, patients were divided into two groups. (A) Allergic fungal sinusitis. (B) Non-allergic fungal sinusitis.

Data was analyzed on a computer using SPSS version 22.0. Descriptive statistics such as frequency and percentage were calculated for categorical variables such as AFS, gender, and comorbidities such as asthma, diabetes, clinical symptoms such as unilateral or bilateral nasal polyps, nasal septal deviation, turbinate hypertrophy, exophthalmos, double vision. Different surgical approaches used and final histopathology, culture results. For quantitative variables such as age, standard deviation and mean were calculated. Statistical significance testing is not applicable to descriptive studies. The onset of this disease was documented.

\section{RESULTS}

Of the 110 patients, 65 were male and 45 were female, with a mean age of 1 and ranged from 7 to 80 years. All patients had nasal polyps. The incidence of AFS was approximately $29.1 \%$ and the remaining 78 had a different pathology. In 32 AFRS cases, the median age was 22 years and ranged from 13 to 42 years. The male to female ratio was $1: 1.1$ as shown in the table: 1 .

\begin{tabular}{|c|c|c|c|c|}
\hline Gender & AFS (\%) & Total & Non AFS(\%) & Total \\
\hline Male & $18 / 32$ & $56.25 \%$ & $52 / 78$ & $66.7 \%$ \\
\hline Female & $14 / 32$ & $43.75 \%$ & $26 / 78$ & $33.3 \%$ \\
\hline Gender & Mean(yrs) & $\mathrm{SD}^{*}$ & Min.(yrs) & Max.(yrs) \\
\hline Male & 23.1 & \pm 21.32 & 07 & 75 \\
\hline Female & 31.5 & \pm 13.94 & 10 & 42 \\
\hline
\end{tabular}

Major deviation of the nasal septum and bilateral hypertrophy of the inferior turbinate's were observed in 6 $(18.7 \%)$ and $4(12.5 \%)$ patients, respectively. This is not significantly related to AFS as a risk factor. Unilateral nasal polyps were observed in $7(21.9 \%)$ patients and bilateral nasal polyps in 18 (52.3\%). As can be seen in Table 2, exophthalmia was observed in $9(28.1 \%)$ patients and diplopia in 7 (21.9\%) patients.

Clinical demonstration in patients with Allergic fungal sinusitis given in Table-2

\begin{tabular}{|l|l|l|}
\hline Clinical features. & $=\mathrm{n}$ & $\%$ age \\
\hline Bilateral polyps. & 18 & 52.3 \\
\hline Unilateral polyps. & 07 & 21.9 \\
\hline Proptosis & 09 & 28.1 \\
\hline Diplopia. & 07 & 21.9 \\
\hline Turbinate hypertrophy & 06 & 18.7 \\
\hline Deviated nasal septum & 04 & 12.5 \\
\hline
\end{tabular}

Table III: Surgical methods applied in allergic fungal sinusitis

\begin{tabular}{|l|l|l|}
\hline Procedures & $=\mathrm{n}$ & $\% \mathrm{age}$ \\
\hline External fronto-ethmoidectomy & 06 & 18.7 \\
\hline Intranasal Ethmoidectomy & 02 & 6.25 \\
\hline Functional endoscopic sinus surgery. & 19 & 59.4 \\
\hline Combination*. & 02 & 6.25 \\
\hline Functional endoscopic sinus Surgery+Septoplasty. & 03 & 9.37 \\
\hline Total & 32 & 100 \\
\hline
\end{tabular}

$19 / 32(59.4 \%)$ of the cases underwent functional endoscopic surgery of the paranasal sinuses, and in 6 $(18.7 \%)$ external fronto-ethmoidectomy. Two patients underwent nasal ethmoidectomy. Septoplasty and endoscopic sinus surgery were performed in a total of 3 $(9.37 \%)$ cases, Table: 3. Final histopathology was sought.32 out of 110 patients reported non-invasive allergic fungal sinusitis with no evidence of tissue infiltration in the material tested. Aspergillus was detected in 22 (68.75\%) 
cases in culture and no fungal growth was found in 10 $(31.25 \%)$ cases.

The remaining 78 patients had pathology unrelated to AFS, 65 were reported as simple nasal inflammatory polyps, and the remaining 13 patients were diagnosed with invasive fungal sinusitis.

\section{DISCUSSION}

Allergic fungal sinusitis is a separate clinical entity with non-specific symptoms. The incidence of AFS is higher than in the international literature. The incidence of AFS is approximately $7 \%$ of all chronic sinusitis requiring surgery ${ }^{13-14}$. A literature review shows that the prevalence of AFS is higher in temperate regions with relatively high humidity. Allergic fungal sinusitis (AFS) has been increasingly defined over the past 20 years ${ }^{15}$. A local study also found a $14 \%$ prevalence of AFS. AFS was seen in 32 out of 110 patients with nasal polyps, therefore the incidence of AFS was higher in this study $(p<0.05)$. Comparing our results with those of national and international researchers, with a fair distribution from men to women, they were observed more often in adolescents with normal immunity ${ }^{16}$. Cody and colleagues also reported an important finding in this study was that most of the patients were poor and middle class living in Karachi. Bilateral nasal obstruction occurred in $52.3 \%$ of patients. Proptosis and visual disturbances were detected in 16 cases after an ophthalmological examination performed by an ophthalmologist on admission. The disease was unilateral in $21.9 \%$ and bilateral in $52.3 \%$. Patients with nasal polyps had a history of asthma (20\%); Anatomical abnormalities such as DNS, turbinate hypertrophy, but no significant correlation with AFS Goldstein also reported a history of atopy in one third of patients and asthma in one third of AFS. As reported by Yousuf M et al., Nasal polyps were strongly associated with AFS ${ }^{17-18}$. Radiological assessments showed multinuclear occupation with areas of attenuation surrounded by the area of hypodensity in most of our cases. Chronic bone changes can be detected by computed tomography. MRI is also useful in disease progression to see intracranial or intra-orbital spread of the disease, which corresponds to a low T1 signal intensity and a high T2 weighted signal. CT is recommended as the initial method of imaging. Surgery plays an important role in the treatment of AFS ${ }^{18-19}$. McGuirt said: "treatment for sinus aspergillosis is surgery. Surgical treatment is the $1^{\text {st }}$ line management forexclusion of the diseased mucosa as well as ventilation and drainage of the affected sinus. "Surgical approaches should be tailored to individual needs. Endoscopic sinus surgery is superior to traditional surgical methods ${ }^{20-21}$. It is significant to understand that the analysis of AFS is not made or ruled out based on the results of the fungal culture. In cases where external approaches are used, the wound is sutured in two layers, and the sutures are removed a week later. Topical nasal steroids were started at the first postoperative visit and continued for the remaining 3 months and then used as needed. Most patients improved their visual symptoms, headaches, and nasal congestion. After discharge, all patients were called for a follow-up one week later. Most patients did not relapse at the monthly follow-up for 1 year after surgery ${ }^{22-}$ 23. The severity of the disease decreased, but very few patients still complained of a runny nose. Recurrence of nasal polyps was found only in $3(9.4 \%)$ cases.

\section{CONCLUSION}

Allergic fungal sinusitis is not uncommon. In our study, AFS was present in a quarter of the cases. Fungal infection should be considered in all patients with chronic sinusitis. Early diagnosis and appropriate treatment give satisfactory results. Failure to recognize AFS can lead to complications such as blindness and an intracranial abscess; unfortunately, such a situation occurs in our patients. We still admit patients with very advanced disease with exophthalmos and visual disturbances and intracranial complications.

\section{REFERENCES}

1. Dhanani $\mathrm{R}$, Pasha $\mathrm{H}$, Khalid $\mathrm{S}$, Ikram M. Incidence of Allergic Fungal Sinusitis among patients with nasal polyps and role of Lund-Mackay scoring in its diagnosis. LaryngoRhino-Otologie. 2020 May;99(S 02).

2. Dhanani R, Khalid S, Salam B, Pasha HA, Yousuf FH, Ikram $M$. Prevalence of allergic fungal sinusitis among patients with nasal polyposis. JPMA. The Journal of the Pakistan Medical Association. 2021;71(6):1605.

3. Qureshi SR, Siddiqui AH, Mehboob S, Ahmed N, Imtiaz A Prevalence of Allergic Fungal Rhinosinusitis Among Patients with Nasal Polyps. ANNALS OF ABBASI SHAHEED HOSPITAL AND KARACHI MEDICAL \& DENTAL COLLEGE. 2020 Apr 6;25(01):44-50.

4. Alghonaim $\mathrm{Y}$, Alfayez A, Alhedaithy $R$, Alsheikh A, Almalki $M$. Recurrence Pattern and Complication Rate of Allergic Fungal Sinusitis: A 10-Year Tertiary Center Experience. International Journal of Otolaryngology. 2020 Dec 18;2020.

5. Hussein A, Obaid A, Lotfi WT, Mostafa HS. Prevalence, Demographic and environmental factors of allergic fungal sinusitis among chronic sinusitis patients with nasal polyps in western Saudi Arabia. Pan. 2019;9:26-30.

6. Sajjad SM, Suhail Z, Ahmed R. Prevalence of fungal infection in nasal polyposis-A cross-sectional study, conducted at a tertiary care hospital in Karachi. JPMA. 2020 Sep $4 ; 2019$

7. Zia S, Naqvi SU, Ahmed S, Farrukh MS, Sheikh SM. Role of Amphotericin B in Nasal Irrigation for Chronic Rhinosinusitis with Nasal Polyps. J. Coll. Phys. Surg. Pak. 2019 Aug 1;29:732-5.

8. Loftus CA, Soler ZM, Koochakzadeh S, Desiato VM, Yoo F, Nguyen SA, Schlosser RJ. Revision surgery rates in chronic rhinosinusitis with nasal polyps: meta-analysis of risk factors. InInternational forum of allergy \& rhinology 2020 Feb (Vol. 10, No. 2, pp. 199-207).

9. Matsumoto Y, Yokoi H, Kawada M, Fujiwara M, Saito K. Two Cases of Allergic Fungal Sinusitis with Differing Postoperative Course. Case reports in otolaryngology. 2019 Nov 21;2019.

10. Bachert C, Bhattacharyya N, Desrosiers M, Khan AH Reducing Fungal Exposure Critical for Treating Rhinosinusitis with or without Polyps [Response to Letter]. Journal of Asthma and Allergy. 2021;14:393-5.

11. Salamah MA, Alsarraj M, Alsolami N, Hanbazazah K, Alharbi $\mathrm{AM}$, Khalifah $\mathrm{Sr}$ W. Clinical, radiological, and histopathological patterns of allergic fungal sinusitis: a single-center retrospective study. Cureus. 2020 Jul;12(7).

12. Curtis L. Reducing Fungal Exposure Critical for Treating Rhinosinusitis with or without Polyps. Journal of Asthma and Allergy. 2021 Mar 24;14:275-6.

13. Marcus S, Schertzer J, Roland LT, Wise SK, Levy JM, DelGaudio JM. Central compartment atopic disease: 
prevalence of allergy and asthma compared with other subtypes of chronic rhinosinusitis with nasal polyps. InInternational forum of allergy \& rhinology $2020 \mathrm{Feb}$ (Vol. 10, No. 2, pp. 183-189).

14. Alajmi S, Koratum RM, Khan Z, Ahmad S, Jeragh A, Ibrahim $\mathrm{H}$, Joseph L, Varghese S. Allergic fungal sinusitis caused by Exserohilumrostratum and literature review. Mycopathologia. 2019 Feb;184(1):89-96.

15. Tanveer U, Gul A, Aqil S. Incidence and Recurrence of Allergic Fungal Sinusitis at Tertiary Care Facility. Indian Journal of Otolaryngology and Head \& Neck Surgery. 2019 Nov;71(3):1832-6.

16. REHMAN F, KHAN S, HAYAT N, ZAMAN W, KHAN A, AHMAD $H$. Determine the Frequency of Fungal Infection in Patients with Nasal Polyps.

17. Alenzi HL, Al Momen A, Molani F. Pediatric allergic fungal rhinosinusitis with extensive intracranial extension-Case report and literature review. International Journal of Surgery Case Reports. 2020 Sep 12.

18. Tyler MA, Luong AU. Current concepts in the management of allergic fungal rhinosinusitis. Immunology and Allergy Clinics. 2020 May 1;40(2):345-59.
19. Shafick PB, El Bahrawy AT, Abd El Fattah MM, Nasr WF Presentation of Allergic Fungal Rhinosinusitis in Sinonasal polyposis. Zagazig University Medical Journal. 2020 Mar 1;26(2):255-61.

20. Brescia G, Alessandrini L, Giacomelli L, Parrino D, Zanotti C, Tealdo G, Franz L, Carraro V, Barion U, Marioni G. A classification of chronic rhinosinusitis with nasal polyps based on structured histopathology. Histopathology. 2020 Jan;76(2):296-307.

21. Kumar VC, Priyanka KS, Raju KB. A Study on Incidence of Fungal Etiology in Nasal Polyposis in GGH, Kakinada.

22. Iqbal M, Khan SF, Khan S, Ahmad W. Fungal Infection Exacerbate Nasal Polyposis: A study conducted at KGN Teaching Hospital Bannu-KPK.

23. Abed AA, Miteab RT, Majid A. Aetiology and Findings of Recurrent Chronic Rhino Sinusitis with Nasal Polyposis after Functional Endoscopic Sinus Surgery. Indian Journal of Forensic Medicine \& Toxicology. 2021 Jan 1;15(1). 